\title{
Signatures of Carrier-Wave Rabi Flopping in GaAs
}

\author{
O. D. Mücke, T. Tritschler, and M. Wegener \\ Institut für Angewandte Physik, Universität Karlsruhe (TH), Wolfgang-Gaede-Straße 1, 76131 Karlsruhe, Germany
}

\begin{abstract}
U. Morgner and F.X. Kärtner
Institut für Hochfrequenztechnik und Quantenelektronik, Universität Karlsruhe(TH), Engesserstraße 5, 76131, Karlsruhe, Germany
\end{abstract} (Received 13 February 2001; published 12 July 2001)

\begin{abstract}
For excitation of the model semiconductor GaAs with optical pulses which are both extremely short ( $5 \mathrm{fs}$ ) and extremely intense $\left(\approx 10^{12} \mathrm{~W} \mathrm{~cm}^{-2}\right)$, we can meet the condition that the Rabi frequency becomes comparable to the band gap frequency - a highly unusual and previously inaccessible situation. Specifically, in this regime, we observe carrier-wave Rabi flopping, a novel effect of nonlinear optics which has been predicted theoretically and which is related to the failure of the area theorem.
\end{abstract}

DOI: 10.1103/PhysRevLett.87.057401

Illuminating a semiconductor with a constant light intensity can lead to a periodic oscillation of the inversion, a phenomenon which is known as Rabi flopping [1]. Using pulsed excitation, Rabi flopping has been observed on semiconductors [2-8]. These Rabi floppings exhibited periods in the range from $50 \mathrm{fs}$ to $1 \mathrm{ps}$. What happens if the light intensity becomes so high that the period of one Rabi oscillation becomes comparable with that of only one cycle of light, $2.9 \mathrm{fs}=h / E_{\mathrm{g}}$ for the band edge of GaAs? Hughes [9] investigated this question theoretically and predicted a new phenomenon of nonlinear optics: Carrierwave Rabi flopping, which is related to the failure of the area theorem. To the best of our knowledge, these two phenomena have not been observed experimentally on any system, i.e., on atoms, molecules, or solids, so far. In this Letter we present experimental evidence for the model semiconductor GaAs.

Corresponding experiments require both huge intensities and electronic coherence. It is thus favorable to concentrate the energy in a very short pulse, ideally with a duration of only one or two optical cycles. What are the anticipated signatures of carrier-wave Rabi flopping? Figure 1(a) schematically depicts conventional Rabi flopping plotted on the Bloch sphere; i.e., the Rabi period is much larger than the light period. The components $u$ and $v$ correspond to the real and imaginary parts of the optical polarization, respectively, and $w$ is the inversion of the two-level system. In this representation, the optical oscillation corresponds to an orbiting of the Bloch vector parallel to the equatorial plane ( $u v$ plane), the oscillation of the inversion to a motion in the $v w$ plane. For a square-shaped pulse with envelope pulse area $\Theta=2 \pi$ starting from the south pole, i.e., all electrons are in the ground state (valence band), the Bloch vector spirals up to the north pole, i.e., all electrons are in the excited state (conduction band), and back to the south pole. This leads to a modulation of the real part of the optical polarization $u$ [Fig. 1(a)], which is roughly similar to a quantum beating. Thus, the corresponding spectrum of the polarization would exhibit two peaks centered around
PACS numbers: 78.47.+p, 42.50.Md, 42.65.Re

the transition frequency. Figure 1(b) shows results for $\Theta=4 \pi$ and for a much shorter pulse, such that the Rabi period equals the light period. Two related aspects are obvious. First, though $\Theta=4 \pi$, the Bloch vector does not come back to the south pole. In this sense, the usual definition of the envelope pulse area $\Theta$ fails. Hence, also the area theorem of nonlinear optics fails. Despite this failure, we quote $\Theta$ for reference in this Letter. Second, it is obvious that the optical polarization becomes strongly distorted [see $u$ versus time in Fig. 1(b)]. Thus, harmonics are being generated, the most prominent of which, for an inversion symmetric medium, is the third harmonic. For low intensities, this is nothing but the resonantly enhanced third-harmonic generation. For very high intensities, i.e., for carrier-wave Rabi flopping, one expects a double peak structure around the third harmonic of the transition frequency. This is illustrated in Fig. 2. Here a single 5 fs pulse impinges onto a sample, and the light emitted into the forward direction around the third harmonic of the
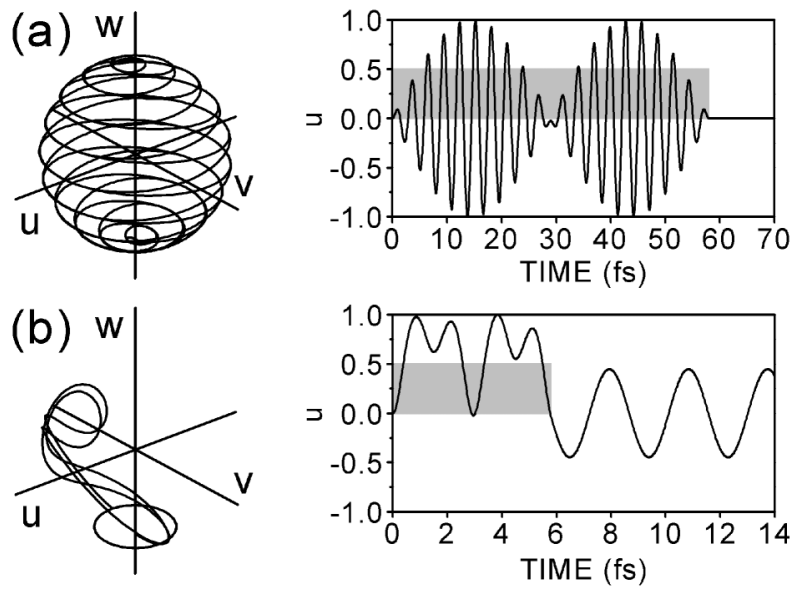

FIG. 1. (a) Scheme of the trace of the Bloch vector for conventional Rabi flopping. Pulse duration is 20 optical cycles, envelope pulse area is $\Theta=2 \pi$. (b) Same for carrier-wave Rabi flopping. Pulse duration is 2 optical cycles, $\Theta=4 \pi$. The optical pulse envelopes are indicated by the grey areas. 


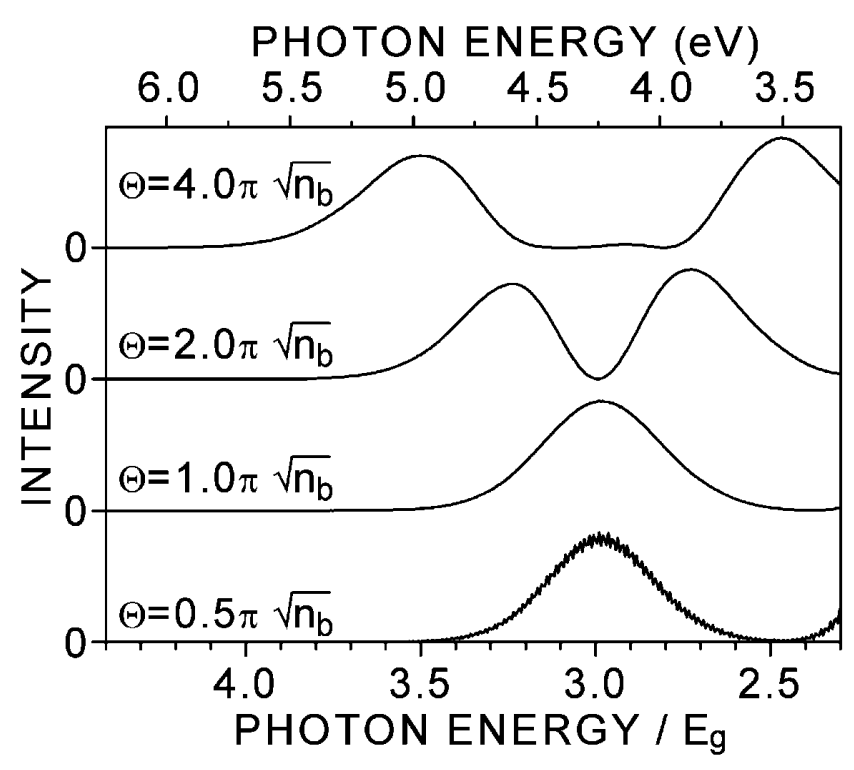

FIG. 2. Theory: Spectra of light emitted into the forward direction around the third harmonic of the transition frequency. The spectra are shown on a linear scale, vertically displaced and individually normalized. Resonant excitation with 5 fs pulses. The envelope pulse area $\Theta$ in front of the sample is indicated. For example, $\Theta=2 \pi \sqrt{n_{\mathrm{b}}}$, with $n_{\mathrm{b}}=\sqrt{\varepsilon_{\mathrm{b}}}$, corresponds to an envelope pulse area of $2 \pi$ inside the sample for vanishing reflection losses.

transition (band edge) frequency is plotted. The envelope pulse area $\Theta$ is parameter. These numerical simulations (Fig. 2), which are meant as a reference point for our experiments, closely follow Refs. [9-11]; i.e., we solve the coupled Maxwell-Bloch equations in one dimension without using the rotating wave approximation and without using the slowly varying envelope approximation. We point out, however, that the actual results are quite different from Refs. $[9,11]$. This is due to the fact that they considered samples much thicker than the wavelength of light while we address "thin" samples [12].

Recently, $5 \mathrm{fs}$ optical pulses at $81 \mathrm{MHz}(=1 / 12 \mathrm{~ns})$ repetition rate have become available [13]. Our homebuilt copy of this laser system very nearly reproduces the pulse properties described in Ref. [13]. Quoted pulse durations, $t_{\text {pulse }}$, refer to the full width at half maximum of the intensity. These linearly polarized pulses are sent into a balanced Michelson interferometer which is actively stabilized by means of the Pancharatnam screw [14]. Using a reflective microscope objective with a numerical aperture of NA $=0.5$, we can tightly focus these pulses to a profile which is roughly Gaussian with $1 \mu \mathrm{m}$ radius. This value has been measured by a knife-edge technique. At the output of the interferometer, each arm has an average power of about $8 \mathrm{~mW}$. The resulting peak intensity of one arm can be estimated as $I_{0}=\frac{8 \mathrm{~mW}}{\pi\left(10^{-4} \mathrm{~cm}\right)^{2}} \frac{12 \mathrm{~ns}}{5 \mathrm{fs}}=0.6 \times$ $10^{12} \mathrm{~W} \mathrm{~cm}^{-2}$. This corresponds to a field envelope $\tilde{E}_{0}=$ $\sqrt{2 \sqrt{\mu_{0} / \varepsilon_{0}} I_{0}}=2.1 \times 10^{7} \mathrm{~V} \mathrm{~cm}^{-1}$. To estimate the envelope pulse area $\Theta$, one furthermore needs the dipole matrix element $d$ of the optical dipole transition. From the literature for GaAs we find $d=0.3 e \mathrm{~nm}$ [15] and $d=0.6 e \mathrm{~nm}$ [5]. Choosing $d=0.5 e \mathrm{~nm}$ in this Letter, this translates into an envelope pulse area $\Theta=\hbar^{-1} d \tilde{E}_{0} \times$ $5 \mathrm{fs}=8.1 \approx 2 \pi$ for one arm, and $\approx 4 \pi$ (two Rabi periods) for two constructively interfering arms of the interferometer. For a 5 fs pulse and a 2.9 fs band gap period, this corresponds to a Rabi frequency which even slightly exceeds the light frequency. It is also interesting to give a very rough estimate for the excited carrier density under these conditions. The GaAs band-to-band absorption coefficient is $\alpha=10^{4} \mathrm{~cm}^{-1}$. If all the light was absorbed according to this number-certainly an upper limitone arrives at a carrier density of $\alpha I_{0} \times 5$ fs $/ 1.42 \mathrm{eV}=$ $1.3 \times 10^{20} \mathrm{~cm}^{-3}$. For constructive interference of the two arms of the interferometer, this number needs to be multiplied by a factor of 4 . Thus, we can safely conclude that the highest carrier densities approach $10^{20} \mathrm{~cm}^{-3}$. In the experiment, we use a $0.6 \mu \mathrm{m}$ thin film of GaAs clad between $\mathrm{Al}_{0.3} \mathrm{Ga}_{0.7} \mathrm{As}$ barriers, grown by metal-organic vapor phase epitaxy on a GaAs substrate. The sample is glued onto a $1 \mathrm{~mm}$ thick sapphire disk and the GaAs substrate is removed. Finally, a $\lambda / 4$-antireflection coating is evaporated. The light emitted by this sample, held under ambient conditions, is collected by a second reflective microscope objective (NA $=0.5)$, is spectrally prefiltered by a sequence of four fused-silica prisms, and is sent into a grating spectrometer connected to a liquid-nitrogen cooled, backilluminated, UV-enhanced charge-coupled-device camera.

Let us first discuss results for single pulses only; i.e., we block one arm of the interferometer. Figure 3 shows spectra at the third harmonic for different pulse intensities $I$ in multiples of $I_{0}$, as defined above. For the attenuation we have used metallic beam splitters on $100 \mu \mathrm{m}$ thin fused silica substrates, the dispersion of which has carefully been compensated for by the extracavity sequence of four $\mathrm{CaF}_{2}$ prisms [13]. The overall behavior resembles that anticipated from the simple modeling (Fig. 2). In particular, we observe the occurrence of two peaks in the third-harmonic spectra at elevated pulse intensities. From the comparison of Fig. 3 with Fig. 2 we conclude that an intensity of $0.218 \times I_{0}$ corresponds to an envelope pulse area of $2 \pi$. At the highest intensity in Fig. $3, I=0.779 \times I_{0}$, the Rabi period is comparable to the light period, and we observe further splitting in the spectrum. This altogether is the anticipated signature of carrier-wave Rabi flopping.

However, using the optical Bloch equations in the context of semiconductors has several shortcomings: (i) For envelope Rabi flopping, it has been shown several times [2-8] within the framework of the semiconductor Bloch equations that the Coulomb interaction of carriers in the bands gives rise to an internal field which adds to the external laser field. This leads to an enhancement of the envelope pulse area $\Theta$ by as much as a factor of 2 [2]. This might explain the discrepancy of a factor of $\sqrt{1 / 0.218}=2.1$ for the envelope pulse area between our previous simple estimate of $\Theta \approx 2 \pi$ for an intensity of 


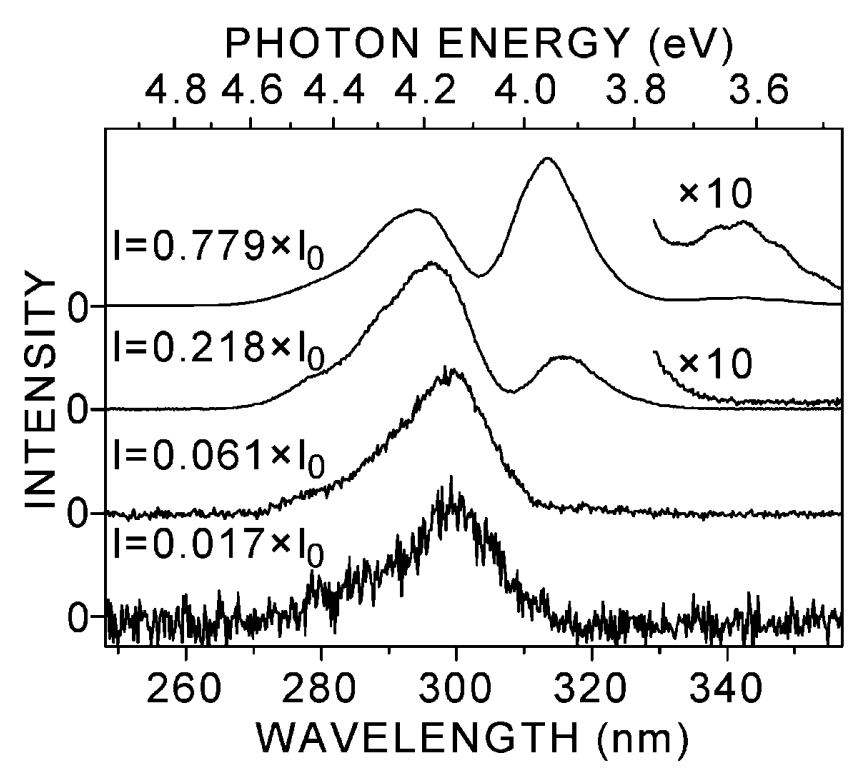

FIG. 3. Experiment: Spectra of light emitted into the forward direction around the third harmonic of the GaAs band gap frequency. The spectra are shown on a linear scale, vertically displaced and individually normalized (from top to bottom: maxima correspond to 5664, 439, 34, and 4 counts/s). Excitation with $5 \mathrm{fs}$ pulses. The intensity $I$ of the pulses is indicated.

$I=I_{0}$, and the comparison of Figs. 2 and 3, which suggests that $I=0.218 \times I_{0}$ corresponds to $\Theta=2 \pi$. (ii) At these large carrier densities, the Coulomb interaction also leads to an appreciable band gap renormalization. Besides sample heating, this can account for the energetic shift between simple model and experiment (compare Figs. 2 and 3). (iii) Also, for carrier densities approaching $10^{20} \mathrm{~cm}^{-3}$, carrier-carrier scattering is the dominant channel of dephasing. On a time scale of only a few femtoseconds, Coulomb quantum kinetic effects arise [16], which cannot be described by a simple dephasing time $T_{2}$. The detailed theoretical description of these truly extreme conditions of light-matter interaction encountered in our experiments is an interesting challenge for many-body theory, but it is far beyond the scope of this experimental work.

In the second set of experiments we study the thirdharmonic spectra for excitation with phase-locked pulse pairs with time delay $\tau$; i.e., we open both arms of the interferometer. It is interesting to note that $\Theta$ is the same for $\tau=0$ and, e.g., for $\tau$ equal to two optical cycles because the two optical fields simply add. Yet, the corresponding Rabi frequency is larger for $\tau=0$. For low intensities [Fig. 4(a)], i.e., for small Rabi frequency as compared to the light frequency, the third-harmonic spectrum is simply modulated as a function of $\tau$ due to trivial interference. In contrast to this, for higher intensities [Figs. 4(b)-4(d)], where the Rabi frequency becomes comparable to the light frequency, the shape of the spectra changes dramatically with time delay $\tau$. For example, for $\tau=0$ in Fig. 4(b), the two pulses simply interfere constructively, and we find the same spectral double maximum structure as in the single pulse experiments
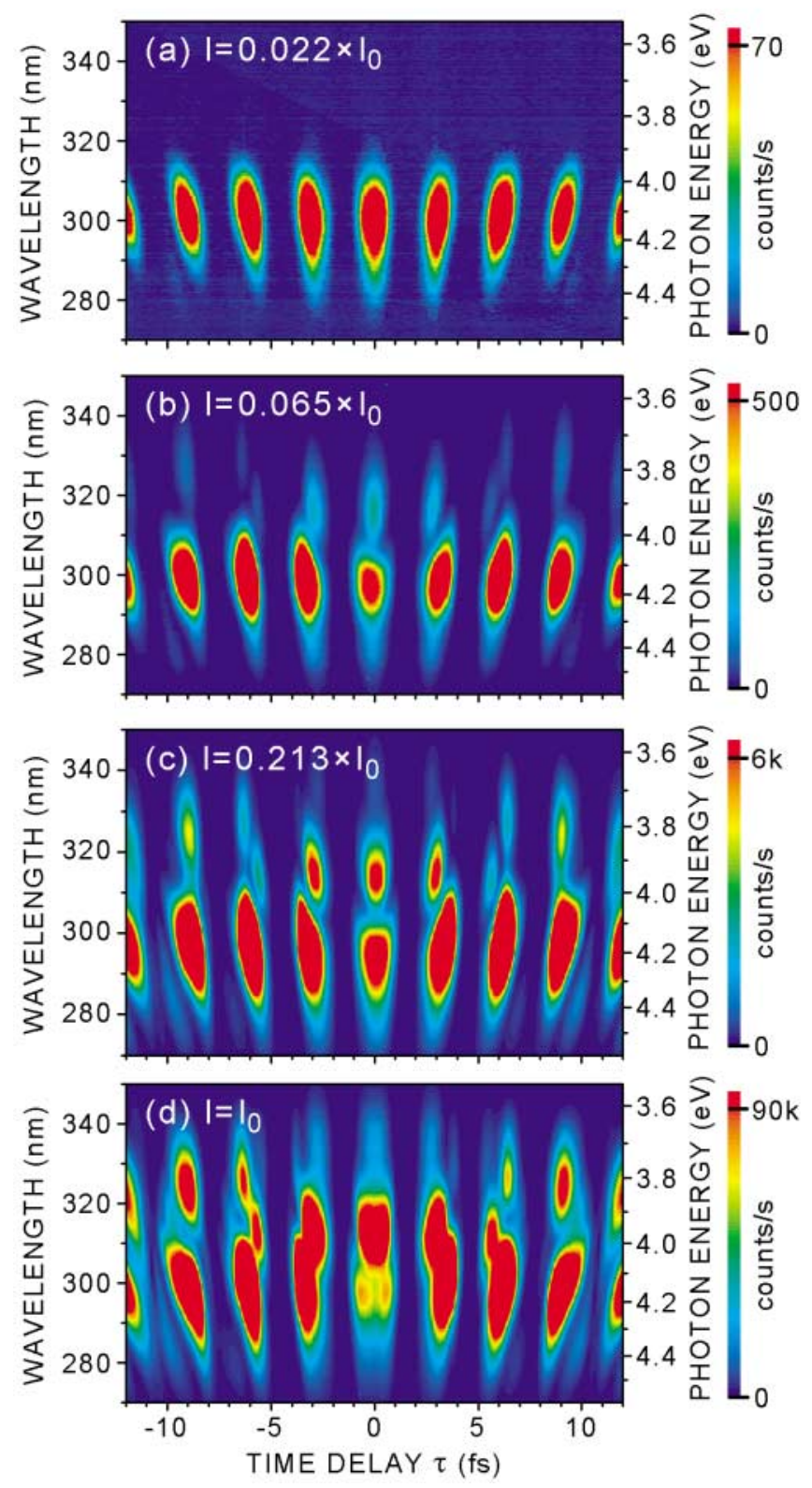

FIG. 4 (color). Experiment: Same as Fig. 3, however, using pairs of phase-locked $5 \mathrm{fs}$ pulses. The signal around the third harmonic of the band gap is depicted versus time delay $\tau$ in a false color plot. (a)-(d) correspond to different intensities $I$ as indicated. I refers to one arm of the interferometer.

(Fig. 3). For larger $\tau$, i.e., after one or two optical cycles, this double maximum disappears and is replaced by one prominent and much larger maximum. For the highest intensity, i.e., for Fig. 4(d) - which corresponds to an envelope pulse area $\Theta$ of more than $4 \pi$ - the behavior is quite involved with additional fine structure for $|\tau|<1$ fs.

In the last set of experiments, we deliberately introduce positive or negative group velocity dispersion by moving one of the extracavity $\mathrm{CaF}_{2}$ prisms in or out of the beam with respect to the optimum position. This leaves the amplitude spectrum of the laser pulses unaffected. Figure 5 shows that one quickly gets out of the regime of carrierwave Rabi flopping: Both the splitting at $\tau=0$ and 

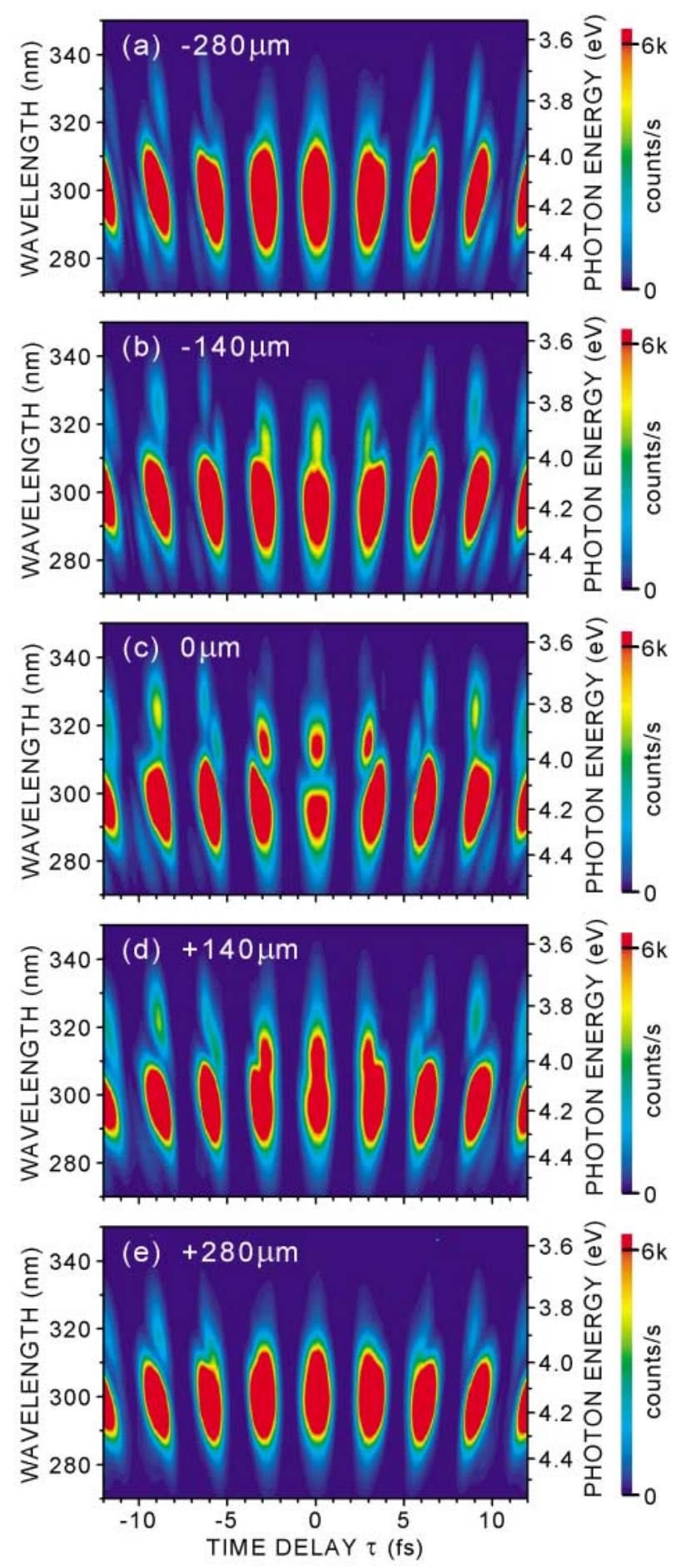

FIG. 5 (color). Experiment: As Fig. 4(c), however, the additional/removed amount of $\mathrm{CaF}_{2}$ material is indicated. This changes the chirp of the pulses. (c) corresponds to Fig. 4(c), i.e., to $I=0.213 \times I_{0}$.

the dependence of the shape on the time delay $\tau$ quickly disappear with increasing pulse chirp. This demonstrates that it is not just the large bandwidth of the pulses but the fact that they are short - two optical cycles - which is important for the observation of carrier-wave Rabi flopping.
In conclusion, we have observed carrier-wave Rabi flopping, which is closely related to the failure of the area theorem of nonlinear optics, for the first time. This unusual regime has become accessible by using both extremely short (5 fs) and extremely intense (estimated Rabi periods $\leq 3 \mathrm{fs}$ ) optical pulses exciting the model semiconductor GaAs, which has a band gap period of $2.9 \mathrm{fs}$. While the single pulse data are reproduced qualitatively by a simple modeling using the Maxwell-Bloch equations without the rotating wave approximation and without the slowly varying envelope approximation, several features of the interferometric data are not reproduced on this level (not depicted). Thus, these experimental data are likely to contain a wealth of information on material dynamics on a time scale of just one or two femtoseconds - a regime, which has previously been completely inaccessible.

The work of M. W. is supported by the DFG Leibniz Award 2000. We thank W. Stolz for the high quality sample.

[1] I. I. Rabi, Phys. Rev. 49, 324 (1936).

[2] R. Binder et al., Phys. Rev. Lett. 65, 899 (1990).

[3] S. T. Cundiff et al., Phys. Rev. Lett. 73, 1178 (1994).

[4] H. Giessen et al., Phys. Rev. Lett. 81, 4260 (1998).

[5] A. Schülzgen et al., Phys. Rev. Lett. 82, 2346 (1999).

[6] L. Bányai et al., Phys. Rev. Lett. 81, 882 (1998).

[7] C. Ciuti et al., Phys. Rev. Lett. 84, 1752 (2000).

[8] M. Saba et al., Phys. Rev. B 62, R16322 (2000).

[9] S. Hughes, Phys. Rev. Lett. 81, 3363 (1998).

[10] R. W. Ziolkowski et al., Phys. Rev. A 52, 3082 (1995).

[11] V. P. Kalosha et al., Phys. Rev. Lett. 83, 544 (1999).

[12] In the modeling using the coupled Maxwell-Bloch equations in one dimension, the parameters for the Bloch part are the following: Transition energy $E_{\mathrm{g}}=1.42 \mathrm{eV}=$ $h /(2.9 \mathrm{fs})$, dipole matrix element $d=0.5 e \mathrm{~nm}$, dephasing time $T_{2}=50 \mathrm{fs}$, and longitudinal relaxation time $T_{1}=\infty$. Excitation with $t_{\text {pulse }}=5 \mathrm{fs}$ pulses, the envelope of which, $\tilde{E}(t)$, is $\operatorname{sech}\left(t / t_{0}\right)$-shaped with $t_{0}=1$ / $[2 \operatorname{arcosh}(\sqrt{2})] t_{\text {pulse }}$. Their center frequency is given by $\hbar \omega_{0}=E_{\mathrm{g}}$. The phase between envelope and carrier wave of the pulses is chosen to be zero; i.e., the actual optical field is given by $E(t)=\tilde{E}(t) \cos \left(\omega_{0} t\right)$. For the Maxwell part, a $0.6 \mu \mathrm{m}$ thin slice of this "material" with $10^{18}$ two-level atoms per $\mathrm{cm}^{3}$ and with additional background dielectric constant $\varepsilon_{\mathrm{b}}=10.9$ is sandwiched between a $\lambda / 4$-antireflection coating (with $\lambda=c_{0} 2.9 \mathrm{fs} / \sqrt[4]{\varepsilon_{\mathrm{b}}}, \quad c_{0}$ is the vacuum velocity of light) on the front side and a semi-infinite substrate with dielectric constant $\varepsilon=\varepsilon_{\mathrm{b}}$ on the back. This corresponds to the sample geometry used in the experiment.

[13] U. Morgner et al., Opt. Lett. 24, 411 (1999).

[14] M. U. Wehner et al., Opt. Lett. 22, 1455 (1997).

[15] N. Peyghambarian et al., Introduction to Semiconductor Optics (Prentice-Hall, Englewood Cliffs, NJ, 1993).

[16] Q. T. Vu et al., Phys. Rev. Lett. 85, 3508 (2000). 